\title{
Detecting structural variants involving repetitive elements: capturing transposition events of IS elements in the genome of Escherichia coli
}

\author{
Heewook Lee ${ }^{1}$, Ellen Popodi ${ }^{2}$, Patricia L Foster ${ }^{2}$, Haixu Tang ${ }^{1 *}$ \\ From Eighth International Society for Computational Biology (ISCB) Student Council Symposium 2012 \\ Long Beach, CA, USA. 13-14 July 2012
}

\section{Background}

Discordant read pairs [1,2] - those deviating either from expected insert size range or correct relative orientation have served as vital clues to identifying structural variants (SV) in genomes. Collecting discordant read pairs is the first step in SV detection and is often done by sequence alignment. When there are repetitive elements, such as insertion sequence (IS), a class of transposable elements in bacterial genomes, discordant read pairs can have multiple mapping loci - making them more challenging to be placed and interpreted. Instead of resolving such tangled mapping results, many tools simply ignore these mapped read pairs, potentially missing SVs involving repetitive elements.

\section{Methods}

We present an idea of using approximate de Bruijn graphs ( $A$-Bruijn graphs) [3] to identify discordant read pairs, in order to discover SVs. Repeats are easily recognized in $A$-Bruijn graphs, as all repetitive elements of the same kind are collapsed into a contiguous edge. When read pairs representing repetitive elements are mapped to a reference $A$-Bruijn graph, only those from novel insertions are flagged as discordant and the rest - those from preexisting insertion loci - mapped concordantly.

We applied this approach to whole genome sequencing data [4] ( 100x per sample using 90bp x 2 paired end Illumina sequencing) obtained from 38 lines of Escherichia coli PFM2, a derivative strain of E. coli K-12 MG1655, and 34 lines of a mismatch repair deficient (deletion of $m u t L$ ) derivative that were propagated for $\sim 3,080$ and $\sim 375$

\footnotetext{
* Correspondence: hatang@indiana.edu

'School of Informatics and Computing, Indiana University, Bloomington, IN, USA

Full list of author information is available at the end of the article
}

generations respectively via a mutation accumulation (MA) strategy. All of the inferred IS insertions were directly confirmed by PCR experiments.

\section{Results}

A total of 27 IS transpositions has been detected and includes 5 out of 12 IS families present in $E$. coli K-12. We have also identified an insertion of IS186 that is fixed among all MA lines and not present in the reference E. coli genome. 24 out 27 inferred insertions were validated by PCR and 3 of them are currently under analysis. The fixed insertion of IS186 in the samples was also confirmed by PCR.

\section{Conclusion}

Our method can pinpoint SVs by identifying discordant read pairs resulting from novel insertions of repetitive elements, where many other currently available tools fail. This result serves as a first step towards inferring the neutral rate of IS transposition in bacterial genomes.

\section{Acknowledgements \\ We thank Indiana University, the entire Foster Lab, and MURI award W911NF-09-1-0444 to P. L. Foster, M. Lynch, H. Tang, and S. Finkel for support.}

\section{Author details}

${ }^{1}$ School of Informatics and Computing, Indiana University, Bloomington, IN, USA. ${ }^{2}$ Department of Biology, Indiana University, Bloomington, IN, USA.

Published: 14 December 2012

\section{References}

1. Raphael BJ, Volik S, Collins C, Pevzner PA: Reconstructing tumor genome architectures. Bioinformatics 2003, 19(Suppl 2):ii162-171.

2. Tuzun E, Sharp AJ, Bailey JA, Kaul R, Morrison VA, Pertz LM, Haugen E, Hayden $\mathrm{H}$, Albertson D, Pinkel D, et al: Fine-scale structural variation of the human genome. Nature genetics 2005, 37(7):727-732.

\section{C) BiolMed Central}


3. Pevzner PA, Tang H, Tesler G: De novo repeat classification and fragment assembly. Genome research 2004, 14(9):1786-1796.

4. Lee H, Popodi E, Tang H, Foster PL: Rate and molecular spectrum of spontaneous mutations in the bacterium Escherichia coli as determined by whole-genome sequencing. Proc Natl Acad Sci 2012, 109(41): E2774-E2783.

doi:10.1186/1471-2105-13-S18-A12

Cite this article as: Lee et al.: Detecting structural variants involving repetitive elements: capturing transposition events of IS elements in the genome of Escherichia coli. BMC Bioinformatics 2012 13(Suppl 18):A12.

Submit your next manuscript to BioMed Central and take full advantage of:

- Convenient online submission

- Thorough peer review

- No space constraints or color figure charges

- Immediate publication on acceptance

- Inclusion in PubMed, CAS, Scopus and Google Scholar

- Research which is freely available for redistribution

Submit your manuscript at www.biomedcentral.com/submit
Ciomed Central 